\title{
(I)-envelopes of unit balls and James' characterization of reflexivity
}

by

\author{
OndŘEJ F. K. Kalenda (Praha)
}

\begin{abstract}
We study the (I)-envelopes of the unit balls of Banach spaces. We show, in particular, that any nonreflexive space can be renormed in such a way that the (I)-envelope of the unit ball is not the whole bidual unit ball. Further, we give a simpler proof of James' characterization of reflexivity in the nonseparable case. We also study the spaces in which the (I)-envelope of the unit ball adds nothing.
\end{abstract}

1. Introduction. The present paper is a continuation of [13] where the (I)-envelopes of sets in Banach spaces were introduced and studied with the aim of using the result of [8] to obtain an easier proof of James' characterization of weak compactness in nonseparable spaces. It was shown there that an easy proof can be given in a large class of spaces. However, an example was exhibited showing that the general situation is not so easy ([13, Example 4.1]).

In this paper we study the (I)-envelopes of the unit balls and in particular two extreme classes of spaces: those where the (I)-envelope adds nothing (Section 4) and those where the (I)-envelope is as large as it can be (Sections 2 and 3). In Section 3 we give a simpler proof of James' characterization of reflexivity using recent results of [15].

Let us start by recalling the definition of the (I)-envelope and its basic properties.

Let $X$ be a Banach space and $B \subset X^{*}$. The $(I)$-envelope of $B$ is defined by the formula

$$
(\mathrm{I})-\operatorname{env}(B)=\bigcap\left\{\overline{\operatorname{conv} \bigcup_{n=1}^{\infty} \overline{\operatorname{conv} C_{n}} w^{*}}: B=\bigcup_{n=1}^{\infty} C_{n}\right\} .
$$

2000 Mathematics Subject Classification: Primary 46B26; Secondary 46A55, 46B10.

Key words and phrases: (I)-envelope, real-valued measurable cardinal, Grothendieck property, reflexive space, James theorem.

This work is part of the research project MSM 0021620839 financed by MSMT and partly supported by the research grant GA ČR 201/06/0018 and by Universidad Politécnica de Valencia. 
The motivation for this definition was the result [8, Theorem 2.3] stating (in the above notation) that $K=(\mathrm{I})-\operatorname{env}(B)$ whenever $K$ is a convex weak* compact set in $X^{*}$ and $B$ a boundary of $K$ (i.e., any element of $X$ attains its maximum on $K$ at some point of $B$ ). We will deal with the (I)-envelopes of closed convex sets in $X$ considered canonically embedded into the bidual. For this case we have the following characterization ([13, Lemma 2.3]).

Lemma 1.1. Let $X$ be a Banach space, $B \subset X$ a closed convex set and $G \in \bar{B}^{w^{*}}$. Then the following assertions are equivalent.

(1) $G \notin(\mathrm{I})-\operatorname{env}(B)$.

(2) There is a sequence of $\xi_{n} \in B_{X^{*}}$ such that

$$
\sup _{x \in B} \limsup _{n \rightarrow \infty} \xi_{n}(x)<\inf _{n \in \mathbb{N}} G\left(\xi_{n}\right) .
$$

(3) There is a sequence of $\xi_{n} \in B_{X^{*}}$ such that

$$
\sup _{x \in B} \limsup _{n \rightarrow \infty} \xi_{n}(x)<\liminf _{n \rightarrow \infty} G\left(\xi_{n}\right) .
$$

(4) There is a sequence of $\xi_{n} \in B_{X^{*}}$ such that

$$
\sup _{x \in B} \limsup _{n \rightarrow \infty} \xi_{n}(x)<\limsup _{n \rightarrow \infty} G\left(\xi_{n}\right) .
$$

Note that in all the conditions (2)-(4) we can replace the assumption that $\xi_{n} \in B_{X^{*}}$ by the assumption that the sequence $\xi_{n}$ is bounded. As an easy consequence of this lemma we obtain the following one.

Lemma 1.2. Let $X$ be a Banach space. Then the (I)-envelope of $X$ in $X^{* *}$ consists exactly of the weak* sequentially continuous elements of $X^{* *}$.

Proof. Let $\xi_{n}$ be a bounded sequence in $X^{*}$. Then

$$
\sup _{x \in X} \limsup _{n \rightarrow \infty} \xi_{n}(x)= \begin{cases}0 & \text { if } \xi_{n} \text { weak }^{*} \text { converges to } 0, \\ +\infty & \text { otherwise. }\end{cases}
$$

The statement now follows easily from Lemma 1.1.

2. Grothendieck spaces and reflexive spaces. A Banach space $X$ is a Grothendieck space if weak and weak* convergences of sequences coincide in $X^{*}$. Clearly, any reflexive space is Grothendieck. There are also nonreflexive Grothendieck spaces: a classical example is the space $\ell_{\infty}$ (a result of Grothendieck [10], see also [4, Theorem VII.15]). Further examples can be found for example in $[1,20,2,17]$.

It is easy to see that $X$ is a Grothendieck space if and only if all elements of $X^{* *}$ are weak* sequentially continuous. Therefore the following theorem is an immediate consequence of Lemma 1.2.

TheOREM 2.1. A Banach space $X$ is Grothendieck if and only if (I) $-\operatorname{env}(X)=X^{* *}$. 
While this theorem is quite easy, the situation with the (I)-envelopes of unit balls is more complicated. It is proved in [13, Example 4.1] that the (I)-envelope of the unit ball of $\ell_{\infty}$ is the whole bidual unit ball. It is asked there whether the same holds for any Grothendieck space. The next theorem shows that this is not the case. Namely, the only spaces for which (I)-env $\left(B_{X}\right)=B_{X^{* *}}$ for any equivalent norm are the reflexive ones.

THEOREM 2.2. Let $X$ be a nonreflexive Banach space. Then there is an equivalent norm $|\cdot|$ on $X$ such that the $(I)$-envelope of the unit ball of $(X,|\cdot|)$ is a proper subset of the respective bidual unit ball.

Proof. If $X$ is separable, then (I)-env $\left(B_{X}\right)=B_{X}($ see [13, Remark 1.1(2)]), hence there is no need to renorm.

Suppose that $X$ is nonseparable. Let $Y \subset X$ be a separable subspace which is not reflexive. (This is possible as reflexivity is separably determined, see e.g. [7, Exercise 4.24].)

Choose some $\xi \in S_{X^{*}}$ such that $\left.\xi\right|_{Y}=0$ and find $x_{0} \in X$ with $\xi\left(x_{0}\right)=1$. Notice that $\left\|x_{0}\right\| \geq 1$. Set

$$
B=\left\{x \in X:\left\|x-\xi(x) x_{0}\right\| \leq 1 \text { and }|\xi(x)|+\operatorname{dist}\left(x-\xi(x) x_{0}, Y\right) \leq 1\right\} .
$$

Then $B$ is clearly a closed absolutely convex set and it is easy to check that

$$
\frac{1}{2+\left\|x_{0}\right\|} B_{X} \subset B \subset\left\|x_{0}\right\| B_{X},
$$

hence $B$ is the closed unit ball of an equivalent norm on $X$. We will show that $\bar{B}^{w^{*}} \backslash(\mathrm{I})-\operatorname{env}(B) \neq \emptyset$.

As $Y \subset X$, the bidual $Y^{* *}$ can be canonically identified with a subspace of $X^{* *}$, namely with the weak* closure of $Y$ in $X^{* *}$. Hence we can take some $F \in S_{Y^{* *}} \backslash X$. Let $Z$ be the span of $Y$ and $x_{0}$. Then $Z$ is separable and $F \in Z^{* *} \backslash Z$, hence $\left.F\right|_{B_{Z^{*}}}$ is not weak ${ }^{*}$ sequentially continuous. It follows that there are $\widetilde{\xi}_{n} \in B_{Z^{*}}$ weak ${ }^{*}$ converging to 0 and $c>0$ such that $F\left(\widetilde{\xi}_{n}\right) \geq c$ for each $n \in \mathbb{N}$. By the Hahn-Banach theorem extend $\widetilde{\xi}_{n}$ to $\xi_{n} \in B_{X^{*}}$.

Set $G=x_{0}+F$. Then $G \in \bar{B}^{w^{*}}$. Indeed, choose a net $y_{\nu} \in S_{Y}$ weak* converging to $F$. Then $x_{0}+y_{\nu}$ weak* converges to $G$. Moreover, $x_{0}+y_{\nu} \in B$ as

$$
\begin{aligned}
\xi\left(x_{0}+y_{\nu}\right) & =1 \\
\left\|x_{0}+y_{\nu}-\xi\left(x_{0}+y_{\nu}\right) x_{0}\right\| & =\left\|y_{\nu}\right\| \leq 1 \\
\operatorname{dist}\left(x_{0}+y_{\nu}-\xi\left(x_{0}+y_{\nu}\right) x_{0}, Y\right) & =\operatorname{dist}\left(y_{\nu}, Y\right)=0 .
\end{aligned}
$$

Further,

$$
\begin{aligned}
\liminf _{n \rightarrow \infty} G\left(\xi+\xi_{n}\right) & =\liminf _{n \rightarrow \infty}\left(\xi\left(x_{0}\right)+\xi_{n}\left(x_{0}\right)+F(\xi)+F\left(\xi_{n}\right)\right) \\
& =1+\lim _{n \rightarrow \infty} \xi_{n}\left(x_{0}\right)+\liminf _{n \rightarrow \infty} F\left(\xi_{n}\right) \geq 1+c
\end{aligned}
$$


We have used here the facts that $F(\xi)=0$ (as $F \in \bar{Y}^{w^{*}}$ and $\left.\xi\right|_{Y}=0$ ) and that $\xi_{n}\left(x_{0}\right) \rightarrow 0\left(\right.$ as $\left.x_{0} \in Z\right)$.

On the other hand, if $x \in B$ and $y \in Y$ is arbitrary, we have

$$
\begin{aligned}
\limsup _{n \rightarrow \infty} & \left(\xi+\xi_{n}\right)(x) \\
& =\xi(x)+\limsup _{n \rightarrow \infty}\left(\xi_{n}\left(x-\xi(x) x_{0}-y\right)+\xi_{n}\left(x_{0}\right) \xi(x)+\xi_{n}(y)\right) \\
& =\xi(x)+\limsup _{n \rightarrow \infty} \xi_{n}\left(x-\xi(x) x_{0}-y\right)+\lim _{n \rightarrow \infty}\left(\xi_{n}\left(x_{0}\right) \xi(x)+\xi_{n}(y)\right) \\
& \leq \xi(x)+\left\|x-\xi(x) x_{0}-y\right\| .
\end{aligned}
$$

(We have used the fact that $\xi_{n}(z) \rightarrow 0$ for all $z \in Z$.) As $y \in Y$ is arbitrary, we get

$$
\limsup _{n \rightarrow \infty}\left(\xi+\xi_{n}\right)(x) \leq \xi(x)+\operatorname{dist}\left(x-\xi(x) x_{0}, Y\right) \leq 1
$$

for all $x \in B$. Hence, by Lemma 1.1 the sequence $\xi+\xi_{n}$ witnesses that $G$ does not belong to the (I)-envelope of $B$.

As a corollary we get the following example.

EXAMPLE 2.3. There is an equivalent norm on $\ell_{\infty}$ such that the $(I)$ envelope of the corresponding unit ball is not the whole bidual unit ball.

We can also give an explicit description of an equivalent norm on $\ell_{\infty}$ with the property described in the above example. Its unit ball is

$$
\left\{x \in \ell_{\infty}:\|x\|_{\infty} \leq 1 \text { and }\left|x_{1}\right|+\limsup _{n \rightarrow \infty}\left|x_{n}\right| \leq 1\right\} .
$$

This example, together with [13, Example 4.1], shows that "(I)-env $\left(B_{X}\right)$ $=B_{X^{* *}}$ " is not an isomorphic property.

The following questions seems to be open.

QUeSTION 2.4. Let $X$ be a Grothendieck space. Is there an equivalent norm on $X$ such that the (I)-envelope of the corresponding unit ball is the whole bidual unit ball?

QUESTION 2.5. Is there a nonreflexive Grothendieck space $X$ such that $(\mathrm{I})-\operatorname{env}\left(B_{X}\right)=B_{X}$ ?

Of course, we cannot have positive answers to both questions. Using [13, Example 4.1] it is easy to see that if Question 2.5 has a positive answer, the corresponding space $X$ cannot contain an isomorphic copy of $\ell_{\infty}$. Hence a natural candidate for such an example is the space from [11].

\section{Towards an easy proof of James' characterization of reflex-} ivity. In this section we discuss the possibility to give an easy proof of the famous James theorem [12, Theorem 5] saying that a Banach space $X$ is reflexive provided any element of $X^{*}$ attains its norm at some point 
of $B_{X}$. For separable spaces there are easy proofs: see e.g. [3, Theorem 3.2] or [9, Theorem 5.7].

Up to our knowledge there is no easy proof of the general case. James' original proof can be divided into two steps. Suppose $X$ is not reflexive. First, one can show that there is a sequence $a_{n}$ in $X$ such that

$$
\inf _{n \in \mathbb{N}} \operatorname{dist}\left(\operatorname{span}\left\{a_{i}: i<n\right\}, \operatorname{conv}\left\{a_{i}: i \geq n\right\}\right)>0 .
$$

The second step is a construction of a functional not attaining its norm starting from such a sequence. Recently in [15] a new proof appeared for the second step in the general nonseparable case. Let us recall the basic steps of that proof (for details see [15]).

We first introduce some notions used in [15]. Let $x_{n}$ be a sequence in a Banach space. A sequence $b_{n}$ is a block sequence of $x_{n}$ if there is a sequence $F_{n}$ of pairwise disjoint finite subsets of $\mathbb{N}$ and a sequence $\lambda_{i}$ of real numbers such that $b_{n}=\sum_{i \in F_{n}} \lambda_{i} x_{i}$ for each $n \in \mathbb{N}$. If $\sum_{i \in F_{n}}\left|\lambda_{i}\right|=1$ for each $n \in \mathbb{N}$, then $b_{n}$ is called a normalized block sequence. If moreover $\lambda_{i} \geq 0$ for all $i$, then $b_{n}$ is a convex block sequence.

(1) If $B_{X^{*}}$ is weak* convex block compact, i.e. any sequence in $B_{X^{*}}$ has a weak* convergent convex block sequence, then the second step of the proof of the James theorem follows from the Simons inequality [21]. This is contained in [15, Lemma 1 and Corollary 2].

(2) If $B_{X^{*}}$ is not weak* sequentially compact but is weak* block compact (i.e. any sequence in $B_{X^{*}}$ has a weak* convergent normalized block sequence), then the second step of the proof can be replaced by an application of the Rosenthal $\ell_{1}$-theorem and the Simons inequality. This is proved in $[15$, Section 2.2].

(3) If $B_{X^{*}}$ is not weak* block compact, then $X$ contains an asymptotically isometric copy of $\ell_{1}$, i.e. there is sequence $a_{n}$ in $B_{X}$ and a sequence of numbers $\delta_{n} \in(0,1)$ converging to 1 such that

$$
\left\|\sum_{i=1}^{n} \lambda_{i} a_{i}\right\| \geq \sum_{i=1}^{n} \delta_{i}\left|\lambda_{i}\right|
$$

for each $n \in \mathbb{N}$ and each $n$-tuple $\lambda_{1}, \ldots, \lambda_{n}$ of real numbers. This is $[15$, Theorem 2].

(4) If $X$ contains an asymptotically isometric copy of $\ell_{1}$, then there is an element of $X^{*}$ which does not attain its norm. This is the content of $[15$, Theorem 3].

We show here an argument replacing the first two steps. Moreover, our argument proves not only the second step but yields a complete proof. The key ingredient is the following proposition. 
Proposition 3.1. Let $X$ be a Grothendieck space such that the dual unit ball is weak* block compact. Then $X$ is reflexive.

Proof. Suppose that $X$ is not reflexive. Then $B_{X^{*}}$ is not weakly compact and hence it is not weakly sequentially compact by the Eberlein-Šmulian theorem [7, Theorem 3.59]. By the Grothendieck property it follows that $B_{X^{*}}$ is not weak* sequentially compact. Let $\xi_{n}$ be a sequence in $B_{X^{*}}$ without a weak* converging subsequence. By the Rosenthal $\ell_{1}$-theorem [18] there is a subsequence of $\xi_{n}$ equivalent to the standard basis of $\ell_{1}$. (Indeed, if $\xi_{n}$ has no such subsequence, it contains a weakly Cauchy subsequence. Further, any weakly Cauchy sequence $\zeta_{n}$ in $X^{*}$ converges in $\left(X^{* * *}, w^{*}\right)$ to a point $\Xi \in X^{* * *}$. Then clearly $\zeta_{n}$ converges in $\left(X^{*}, w^{*}\right)$ to $\left.\Xi\right|_{X}$, hence is weak* convergent.) Hence we can suppose that the sequence $\xi_{n}$ itself is equivalent to the basis of $\ell_{1}$, i.e. there is some constant $c>0$ such that

$$
\left\|\sum_{i=1}^{n} \lambda_{i} \xi_{i}\right\| \geq c \sum_{i=1}^{n}\left|\lambda_{i}\right|
$$

for all $n \in \mathbb{N}$ and all $n$-tuples $\lambda_{1}, \ldots, \lambda_{n}$ of real numbers. In particular the space

$$
Y=\overline{\operatorname{span}\left\{\xi_{n}: n \in \mathbb{N}\right\}}
$$

is isomorphic to $\ell_{1}$. We claim that $\xi_{n}$ has no weak* convergent normalized block sequence. Suppose that $\zeta_{n}$ is such a sequence. Then $\frac{1}{2}\left(\zeta_{2 n}-\zeta_{2 n+1}\right)$ is a normalized block sequence of $\xi_{n}$ weak* converging to 0 , hence we can without loss of generality suppose that $\zeta_{n}$ weak* converges to 0 . (This easy remark is made in [15, Section 1.2].)

Now, by the Grothendieck property $\zeta_{n}$ weakly converges to 0 . As all $\zeta_{n}$ belong to $Y$ and $Y$ is isomorphic to $\ell_{1}$, it follows from the Schur theorem [7, Theorem 5.19] that $\zeta_{n}$ norm converges to 0 . However, as $\zeta_{n}$ is a normalized block sequence of $\xi_{n}$, we get $\left\|\zeta_{n}\right\| \geq c$ for all $n \in \mathbb{N}$. This is a contradiction completing the proof.

This proposition enables us to give the following proof of the James theorem.

A proof of James' characterization of reflexivity. Let $X$ be a Banach space such that every element of $X^{*}$ attains its norm at some point of $B_{X}$. By [8, Theorem 2.3], the (I)-envelope of $B_{X}$ is the whole $B_{X^{* *}}$. By [16, Theorem 1.4] or [13, Theorem 3.4], $X$ has the Grothendieck property. Suppose $X$ is not reflexive. By Proposition $3.1, B_{X^{*}}$ is not weak* block compact. By [15, Theorem 2], $X$ contains an asymptotically isometric copy of $\ell_{1}$ and hence, by [15, Theorem 3], there is an element of $X^{*}$ not attaining its norm. This completes the proof. 
The proof just given is quite short but uses a large number of nontrivial results. Nonetheless, it seems to the author that this proof makes the James theorem more accessible. However, if we want an easy proof, we should give an easy proof of the fact that $X$ contains an asymptotically isometric copy of $\ell_{1}$ whenever $X$ is a nonreflexive space with (I)-env $\left(B_{X}\right)=B_{X^{* *}}$. Let us briefly discuss what would be needed for that.

In $[15$, Section 3] the following quantities are defined for any bounded sequence $\xi_{n}$ in $X^{*}$ :

$$
\begin{aligned}
& \delta\left(\xi_{n}\right)_{n}=\sup _{x \in B_{X}} \limsup _{n \rightarrow \infty} \xi_{n}(x), \\
& \varepsilon\left(\xi_{n}\right)_{n}=\inf \left\{\delta\left(\zeta_{n}\right)_{n}:\left(\zeta_{n}\right)_{n} \text { is a normalized block sequence of }\left(\xi_{n}\right)_{n}\right\} .
\end{aligned}
$$

The main part of the proof of [15, Theorem 2] is a construction of an asymptotically isometric copy of $\ell_{1}$ in $X$ starting from a bounded sequence $\xi_{n}$ in $X^{*}$ satisfying

$$
\varepsilon\left(\xi_{n}\right)_{n}=\delta\left(\xi_{n}\right)_{n}>0 .
$$

Such a sequence $\xi_{n}$ can be constructed by the diagonal method ([15, Lemma 3]) from any sequence $\xi_{n}^{0}$ such that $\delta\left(\zeta_{n}\right)_{n}>0$ for any normalized block sequence $\zeta_{n}$ of $\xi_{n}^{0}$.

Now, if (I)-env $\left(B_{X}\right)=B_{X^{* *}}$, then (by Lemma 1.1)

$$
\delta\left(\xi_{n}\right)_{n}=\sup _{F \in B_{X^{* *}}} \limsup _{n \rightarrow \infty} F\left(\xi_{n}\right)
$$

for each bounded sequence $\xi_{n}$ in $X^{*}$. Note that the right-hand side is zero if and only if $\xi_{n}$ weakly converges to 0 .

Hence we would need an easy proof of the following fact.

FACT. Let $X$ be a Banach space such that $(\mathrm{I})-\operatorname{env}\left(B_{X}\right)=B_{X^{* *}}$ and $B_{X^{*}}$ is weakly block compact. Then $X$ is reflexive.

This fact follows from Rosenthal's and Schur's theorems. In fact, we have the following characterization of spaces not containing $\ell_{1}$ :

Proposition 3.2. A Banach space $Y$ contains no isomorphic copy of $\ell_{1}$ if and only if $B_{Y}$ is weakly block compact.

Proof. Suppose that $Y$ contains no isomorphic copy of $\ell_{1}$ and $y_{n}$ is a sequence in $B_{Y}$. By the Rosenthal theorem [18] there is a subsequence $y_{n_{k}}$ which is weakly Cauchy. Now, $\frac{1}{2}\left(y_{n_{2 k}}-y_{n_{2 k+1}}\right)$ is a normalized block sequence of $y_{n}$ weakly converging to 0 . Hence $B_{Y}$ is weakly block compact.

Conversely, let $y_{n}$ be a sequence in $B_{Y}$ equivalent to the standard basis of $\ell_{1}$. Let $z_{n}$ be a normalized block sequence of $y_{n}$ weakly converging to 0 . By the Schur theorem $z_{n}$ norm converges to 0 , which is impossible for any normalized block sequence of $y_{n}$. 
Hence, the proof of the above Fact goes as follows: If $B_{X^{*}}$ is weakly block compact, $X^{*}$ contains no copy of $\ell_{1}$. Now, in this case $B_{X^{*}}$ is weak* sequentially compact by the Rosenthal theorem. If, moreover, (I)-env $\left(B_{X}\right)$ $=B_{X^{* *}}$, we find that $B_{X^{*}}$ is weakly sequentially compact and hence $X$ is reflexive.

This argument is essentially the same as the one given above. The author does not know whether one can give an essentially simpler proof of the Fact. Neither is it clear whether one could give a comparably simple proof of the James characterization of weak compactness.

4. Spaces in which the (I)-envelope adds nothing. In this section we study the other extreme - the Banach spaces $X$ for which (I)-env $(X)=X$ or at least (I)-env $\left(B_{X}\right)=B_{X}$. As an immediate consequence of Lemma 1.2 we get the following theorem.

Theorem 4.1. Let $X$ be a Banach space. Then $(\mathrm{I})-\operatorname{env}(X)=X$ if and only if the only weak* sequentially continuous elements of $X^{* *}$ are those from $X$.

Note, that weak* continuous elements of $X^{* *}$ belong to $X$ by [7, Theorem 3.16]. Hence the condition in the above theorem means that weak* sequential continuity of a linear function on $X^{*}$ implies its weak* continuity. It is the case for example if $\left(B_{X^{*}}, w^{*}\right)$ is angelic (cf. [13, Theorem 3.1]) or, more generally, if $\left(B_{X^{*}}, w^{*}\right)$ is sequential (i.e., sequentially closed sets are closed). We will see below that these sufficient conditions are not necessary.

If (I)-env $(X)=X$, then (I) $\operatorname{env}(B)=B$ for all closed convex subsets $B \subset X$, in particular (I)-env $\left(B_{X}\right)=B_{X}$. It seems unclear whether the converse is true, i.e. we have the following problem.

Question 4.2. Let $X$ be a Banach space such that $(\mathrm{I})-\operatorname{env}\left(B_{X}\right)=B_{X}$. Is then $(\mathrm{I})-\operatorname{env}(X)=X$ ?

Note that the answer is positive if $B_{X^{*}}$ is weak* sequentially compact. In this case any weak* sequentially continuous element of $B_{X^{* *}}$ belongs to (I)-env $\left(B_{X}\right)$ (see [13, Proposition 2.4]), hence the positive answer follows from Theorem 4.1. Below we show that the answer is also positive for $\ell_{1}(\Gamma)$ spaces. However, the general answer seems to be unknown (cf. also Question 2.5).

We also note that if $(\mathrm{I})-\operatorname{env}\left(B_{X}\right)=B_{X}$, then $(\mathrm{I})-\operatorname{env}(B)=B$ for each bounded closed convex subset of $X$. Hence the class of spaces in which (I)-env $\left(B_{X}\right)=B_{X}$ is stable with respect to isomorphisms and subspaces.

Now we will consider the space $X=\ell_{1}(\Gamma)$ for a set $\Gamma$ with the standard norm. The dual $X^{*}$ is canonically identified with the space $\ell_{\infty}(\Gamma)$ which is isometric to the space $C(\beta \Gamma)$ of continuous functions on the Čech-Stone 
compactification of the discrete space $\Gamma$. The bidual $X^{* *}$ is then, by the Riesz theorem, identified with the space of all finite signed Radon measures on $\beta \Gamma$, which canonically correspond to finite signed finitely additive measures on the set $\Gamma$. We will use the latter description (see [6, Corollary IV.5.3]).

We have the following characterization of the (I)-envelope of the unit ball of $\ell_{1}(\Gamma)$.

Proposition 4.3. Let $X=\ell_{1}(\Gamma)$ and $\mu$ be a finite signed finitely additive measure on $\Gamma$ with $\|\mu\| \leq 1$. The following are equivalent.

(i) $\mu$ is $\sigma$-additive.

(ii) $\mu$ is weak $k^{*}$ sequentially continuous.

(iii) $\mu \in(\mathrm{I})-\operatorname{env}\left(B_{X}\right)$.

Proof. The implication (iii) $\Rightarrow$ (ii) follows from Lemma 1.2, and (i) $\Rightarrow$ (ii) follows from the Lebesgue dominated convergence theorem.

(ii) $\Rightarrow$ (i). Suppose that $\mu$ is not $\sigma$-additive. Then there is a sequence $A_{n}$ of pairwise disjoint sets such that

$$
\mu\left(\bigcup_{n \in \mathbb{N}} A_{n}\right) \neq \sum_{n \in \mathbb{N}} \mu\left(A_{n}\right) .
$$

Let $f_{n}$ be the characteristic function of $A_{1} \cup \cdots \cup A_{n}$ and $f$ be the characteristic function of $\bigcup_{n \in \mathbb{N}} A_{n}$. Then $f_{n} \rightarrow f$ in the weak* topology of $\ell_{\infty}(\Gamma)$ but $\mu\left(f_{n}\right)$ does not converge to $\mu(f)$.

(i) $\Rightarrow$ (iii). As the (I)-envelope of $B_{X}$ is absolutely convex, it is enough to prove the implication for probability measures. Suppose that $\mu$ is a $\sigma$ additive probability on $\Gamma$. Let $B_{n} \nearrow B_{X}$. Set

$$
A_{n}=\left\{\gamma \in \Gamma: e_{\gamma} \in B_{n}\right\}
$$

where $\left\{e_{\gamma}: \gamma \in \Gamma\right\}$ is the canonical basis of $\ell_{1}(\Gamma)$. Then $A_{n} \nearrow \Gamma$ and hence $\mu\left(A_{n}\right) \nearrow 1$ (by $\sigma$-additivity of $\mu$ ).

Let $\varepsilon>0$ be arbitrary. There is some $n$ such that $\mu\left(A_{n}\right)>1-\varepsilon / 2$. As the set $\overline{\operatorname{conv} B_{n}} w^{*}$ clearly contains all probabilities supported by $A_{n}$, we get

$$
\mu_{n}=\frac{\left.\mu\right|_{A_{n}}}{\mu\left(A_{n}\right)} \in \overline{\operatorname{conv} B_{n}} w^{*} .
$$

Further,

$$
\left\|\mu-\mu_{n}\right\|=\mu\left(\Gamma \backslash A_{n}\right)+\left(1-\mu\left(A_{n}\right)\right)<\varepsilon / 2+\varepsilon / 2=\varepsilon .
$$

As $\varepsilon>0$ is arbitrary, we get

$$
\mu \in \overline{\bigcup_{n \in \mathbb{N}} \overline{\operatorname{conv} B_{n}} w^{*}\|\cdot\|} .
$$

As $B_{n} \nearrow B_{X}$ are arbitrary, we get $\mu \in(\mathrm{I})-\operatorname{env}\left(B_{X}\right)$.

From the above proposition we derive the following theorem. 
TheORem 4.4. Let $\Gamma$ be any set. The following are equivalent.

(a) No cardinal number at most equal to card $\Gamma$ is real-valued measurable.

(b) (I)-env $\left(B_{\ell_{1}(\Gamma)}\right)=B_{\ell_{1}(\Gamma)}$.

(c) $(\mathrm{I})-\operatorname{env}\left(\ell_{1}(\Gamma)\right)=\ell_{1}(\Gamma)$.

Proof. The implication $(\mathrm{c}) \Rightarrow(\mathrm{b})$ is trivial.

(b) $\Rightarrow$ (a). Suppose that there is some real-valued measurable cardinal number at most equal to card $\Gamma$. This means that there is a $\sigma$-additive probability measure $\mu$ on $\Gamma$ such that $\mu(\{\gamma\})=0$ for all $\gamma \in \Gamma$. By the previous proposition $\mu \in(\mathrm{I})-\operatorname{env}\left(B_{\ell_{1}(\Gamma)}\right)$. Further, clearly $\mu \notin \ell_{1}(\Gamma)$.

(a) $\Rightarrow(\mathrm{c})$. Suppose that $\mu \in(\mathrm{I})-\operatorname{env}\left(\ell_{1}(\Gamma)\right) \backslash \ell_{1}(\Gamma)$. By Lemma 1.2 we see that $\mu$ is weak* sequentially continuous, and so by Proposition 4.3 the measure $\mu /\|\mu\|$ (and hence also $\mu$ ) is $\sigma$-additive. Set

$$
\nu=\sum_{\gamma \in \Gamma} \mu(\{\gamma\}) e_{\gamma} .
$$

Then $\nu \in \ell_{1}(\Gamma)$ and hence $\mu-\nu \neq 0$. So $\mu-\nu$ is a nonzero $\sigma$-additive finite measure on $\Gamma$ which is zero on singletons, hence there is some real-valued measurable cardinal at most equal to card $\Gamma$.

As a consequence we get the following example.

EXAMPLE 4.5.

(a) Let $X=\ell_{1}\left(\left[0, \omega_{1}\right]\right)$. Then $(\mathrm{I})-\operatorname{env}(X)=X$. In this case $\left(B_{X^{*}}, w^{*}\right)$ is not angelic (even not sequential).

(i) Under the continuum hypothesis $B_{X^{*}}$ is not weak $k^{*}$ sequentially compact.

(ii) Under Martin's axiom and negation of the continuum hypothesis $B_{X^{*}}$ is weak* sequentially compact.

(b) Let $X=\ell_{1}([0,1])$. Then $\left(B_{X^{*}}, w^{*}\right)$ is not weak $k^{*}$ sequentially compact.

(i) If there is no real-valued measurable cardinal at most equal to the continuum, then (I)-env $(X)=X$.

(ii) If there is a real-valued measurable cardinal at most equal to the continuum, then (I)-env $\left(B_{X}\right) \supsetneq B_{X}$.

(c) If there are no real-valued measurable cardinals (for example under $V=L)$, then $(\mathrm{I})-\operatorname{env}\left(\ell_{1}(\Gamma)\right)=\ell_{1}(\Gamma)$ for any set $\Gamma$.

Proof. (a) The cardinal $\aleph_{1}$ is not real-valued measurable by [22, §1, Satz (A)]. Therefore (I)-env $(X)=X$. The fact that $B_{X^{*}}$ is not sequential is easy: $\left(B_{X^{*}}, w^{*}\right)$ is homeomorphic to $[-1,1]^{\left[0, \omega_{1}\right]}$ and the set

$$
\left\{x \in[-1,1]^{\left[0, \omega_{1}\right]}:\{\gamma: x(\gamma) \neq 0\} \text { is countable }\right\}
$$


is sequentially closed but not closed. Assertion (i) follows from the wellknown fact that $\{0,1\}^{[0,1]}$ is not sequentially compact. Assertion (ii) follows from the result of Malykhin and Shapirovskiı̌ [14] that under Martin's axiom all compact spaces of cardinality at most equal to the continuum are sequentially compact.

(b) We use again the fact that $\{0,1\}^{[0,1]}$ is not sequentially compact and Theorem 4.4.

Assertion (c) follows from Theorem 4.4. The fact that under $V=L$ there are no real-valued measurable cardinals follows from [22, §2, Satz 3] and [19].

5. Final remarks. All the results of this paper are formulated for real spaces. However, they can be easily transferred to the complex case as well using the method described in [13, Section 5] and the following observations:

- The unit balls constructed in Theorem 2.2 and Example 2.3 are also absolutely convex in the complex case.

- James' characterization of reflexivity of complex spaces follows easily from the real version.

- Propositions 3.1 and 3.2 are true for complex spaces, as also are the Rosenthal and Schur theorems. (This is easy for the Schur theorem, and for the Rosenthal theorem it is proved in [5].)

\section{References}

[1] T. Ando, Convergent sequences of finitely additive measures, Pacific J. Math. 11 (1961), 395-404.

[2] J. Bourgain, $H^{\infty}$ is a Grothendieck space, Studia Math. 75 (1983), 193-216.

[3] R. Deville, G. Godefroy, and V. Zizler, Smoothness and Renormings in Banach Spaces, Pitman Monogr. 64, Longman and Wiley, 1993.

[4] J. Diestel, Sequences and Series in Banach Spaces, Grad. Texts in Math. 92, Springer, New York, 1984.

[5] L. E. Dor, On sequences spanning a complex $\ell_{1}$ space, Proc. Amer. Math. Soc. 47 (1975), 515-516.

[6] N. Dunford and J. T. Schwartz, Linear Operators I, Interscience, New York, 1957.

[7] M. Fabian et al., Functional Analysis and Infinite-Dimensional Geometry, CMS Books Math. 8, Springer, New York, 2001.

[8] V. P. Fonf and J. Lindenstrauss, Boundaries and generation of convex sets, Israel J. Math. 136 (2003), 157-172.

[9] V. P. Fonf, J. Lindenstrauss and R. R. Phelps, Infinite dimensional convexity, in: Handbook of the Geometry of Banach Spaces, Vol. 1, W. B. Johnson and J. Lindenstrauss (eds.), North-Holland, 2001, 599-670.

[10] A. Grothendieck, Sur les applications linéaires faiblement compactes d'espaces du type $C(K)$, Canad. J. Math. 5 (1953), 129-173.

[11] R. Haydon, A non-reflexive Grothendieck space that does not contain $\ell_{\infty}$, Israel J. Math. 40 (1981), 65-73. 
[12] R. C. James, Characterizations of reflexivity, Studia Math. 23 (1963/1964), 205-216.

[13] O. Kalenda, (I)-envelopes of closed convex sets in Banach spaces, Israel J. Math., to appear.

[14] V. I. Malykhin and B. E. Shapirovskiŭ, Martin's axiom and properties of topological spaces, Dokl. Akad. Nauk SSSR 213 (1973), 532-535 (in Russian).

[15] M. Morillon, A new proof of James' sup theorem, Extracta Math. 20 (2005), 261-271.

[16] O. Nygaard, A remark on Rainwater's theorem, Ann. Math. Inform. 32 (2005), $125-127$.

[17] H. Pfitzner, Weak compactness in the dual of a $C^{*}$-algebra is determined commutatively, Math. Ann. 298 (1994), 349-371.

[18] H. P. Rosenthal, A characterization of Banach spaces containing $\ell_{1}$, Proc. Nat. Acad. Sci. U.S.A. 71 (1974), 2411-2413.

[19] D. Scott, Measurable cardinals and constructible sets, Bull. Acad. Polon. Sci. Sér. Sci. Math. Astronom. Phys. 9 (1961), 521-524.

[20] G. L. Seever, Measures on F-spaces, Trans. Amer. Math. Soc. 133 (1968), 267-280.

[21] S. Simons, A convergence theorem with boundary, Pacific J. Math. 40 (1972), 703-708.

[22] S. Ulam, Zur Masstheorie in der allgemeinen Mengenlehre, Fund. Math. 16 (1930), $140-150$.

Department of Mathematical Analysis

Faculty of Mathematics and Physics

Charles University

Sokolovská 83

18675 Praha 8, Czech Republic

E-mail: kalenda@karlin.mff.cuni.cz

Received May 18, 2006

Revised version June 26, 2007 